\title{
STUDY OF ELECTRON PAIR PRODUCTION BELOW THE $Z$ MASS AT THE CERN p̄p COLLIDER
}

\section{The UA2 Collaboration}

Bern - Cambridge - CERN - Dortmund - Heidelberg - Melbourne Milano - Orsay (LAL) - Pavia - Perugia - Pisa - Saclay (CEN)

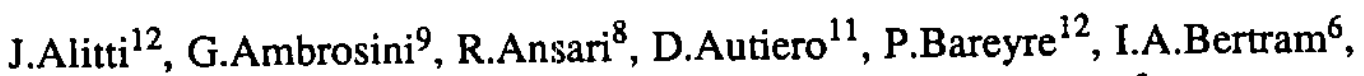
G.Blaylock $^{3, a}$, P.Bonamy ${ }^{12}$, K.Borer $^{1}$, M.Bourliaud ${ }^{12}$, D.Buskulic ${ }^{8}$, G.Carboni $^{11}$, D.Cavalli ${ }^{7}$, V.Cavasinni ${ }^{11}$, P.Cenci ${ }^{10}$, J.C.Chollet ${ }^{8}$, C.Conta ${ }^{9}$, G.Costa ${ }^{7}$, F.Costantini ${ }^{11}$, L.Cozzi ${ }^{7}$, A.Cravero ${ }^{7}$, M.Curatolo ${ }^{11}$, A.Dell'Acqua ${ }^{9}$, T.DelPrete ${ }^{11}$, R.S.DeWolf ${ }^{2}$, L.DiLella ${ }^{3}$, Y.Ducros ${ }^{12}$, G.F.Egan ${ }^{6}$, K.F.Einsweiler ${ }^{3, b}$, B.Esposito ${ }^{11}$, L.Fayard $^{8}$, A.Federspiel ${ }^{1}$, R.Ferrari ${ }^{9}$, M.Fraternali ${ }^{9, c}$, D.Froidevaux ${ }^{3}$, G.Fumagalli ${ }^{9}$, J.M.Gaillard ${ }^{8}$, F.Gianotti ${ }^{7}$, O.Gildemeister ${ }^{3}$, C.Gössling ${ }^{4}$, V.G.Goggi ${ }^{9}$, S.Grünendahl ${ }^{5}$, K.Hara ${ }^{1, d}$, S.Hellman ${ }^{3}$, J.Hrivnac ${ }^{3}$, H.Hufnagel ${ }^{4}$, E.Hugentobler ${ }^{1}$, K.Hultqvist ${ }^{3, e}$, E.Iacopini ${ }^{11, f}$, J.Incandela ${ }^{7}$, K.Jakobs $^{3}$, P.Jenni ${ }^{3}$, E.E.Kluge ${ }^{5}$, N.Kurz ${ }^{5}$, S.Lami ${ }^{11}$, P.Lariccia ${ }^{10}$, M.Lefebvre ${ }^{3}$, L.Linssen ${ }^{3}$, M.Livan ${ }^{9}, \mathrm{~g}$, P.Lubrano $^{3,10}$, C.Magneville ${ }^{12}$, L.Mandelli ${ }^{7}$, L.Mapelli ${ }^{3}$, M.Mazzanti ${ }^{7}$, K.Meier ${ }^{3, h}$,

B.Merkel $^{8}$, J.P.Meyer ${ }^{12}$, M.Moniez ${ }^{8}$, R.Moning ${ }^{1}$, M.Morganti ${ }^{11, i}$, L.Müller ${ }^{1}$,

D.J.Munday ${ }^{2}$, M.Nessi ${ }^{3}$, F.Nessi-Tedaldi ${ }^{3}$, C.Onions ${ }^{3}$, T.Pal ${ }^{1}$, M.A.Parker $^{2}$,

G.Parrour $^{8}$, F.Pastore ${ }^{9}$, E.Pennacchio9, J.M.Pentney ${ }^{3}$, M.Pepe ${ }^{3}$, L.Perini ${ }^{7}$,c,

C.Petridou $^{11}$, P.Petroff ${ }^{8}$, H.Plothow-Besch ${ }^{3}$, G.Polesello ${ }^{3,9}$, A.Poppleton ${ }^{3}$, K.Pretzl $^{1}$, M.Primavera ${ }^{11, j}$, M.Punturo ${ }^{10}$, J.P.Repellin ${ }^{8}$, A.Rimoldi ${ }^{9}$, M.Sacchi ${ }^{9}$, P.Scampoli ${ }^{10}$,

J.Schacher ${ }^{1}$, B.Schmidt ${ }^{4}$, V.Simak ${ }^{3}$, S.L.Singh ${ }^{2}$, V.Sondermann ${ }^{4}$, R.Spiwoks ${ }^{4}$,

S.Stapnes ${ }^{3}$, C.Talamonti ${ }^{10}$, F.Tondini ${ }^{10}$, S.N.Tovey ${ }^{6}$, E.Tsesmelis ${ }^{4}$, G.Unal ${ }^{8}$, M.Valdata-Nappi ${ }^{11, j}$, V.Vercesi ${ }^{9}$, A.R.Weidberg ${ }^{3, k}$, P.S.Wells ${ }^{2, \ell}$, T.O.White ${ }^{2}$, D.R.Wood ${ }^{8}$, S.A.Wotton ${ }^{2, \ell}$, H.Zaccone ${ }^{12}$, A.Zylberstejn ${ }^{12}$ 


\begin{abstract}
Results on the cross section for the production of electron pairs in $\overrightarrow{\mathrm{p} p}$ collisions at $\sqrt{s}_{\mathrm{s}}=630 \mathrm{GeV}$ are presented. The measured value is $\sigma=405 \pm 51$ (stat.) \pm 84 (syst.) pb, in the invariant mass interval $10<\mathrm{m}<70 \mathrm{GeV}$. The results are compared to recent theoretical calculations which include $\mathrm{O}\left(\alpha_{5}^{2}\right) \mathrm{QCD}$ contributions. The comparison of these data with those of lower energy experiments show approximate scaling as a function of the variable $\sqrt{ } \tau=\mathrm{m} / \mathrm{V}_{\mathrm{s}}$.
\end{abstract}

1 Laboratorium für Hochenergiephysik, Universită Bem, Sidlerstraße 5, 3012 Bem, Switzerland

2 Cavendish Laboratory, University of Cambridge, Cambridge, CB3 OHE, UK

3 CERN, 1211 Geneva 23, Switzerland

4 Lehrstuhl für Exp. Physik IV. Universitat Dortmund, 4600 Dortmund, FRG

5 Institut für Hochenergiephysik der Universitătt-Heidelberg, Schróderstraße 90, 6900 Heidelberg, FRG

6 School of Physics, University of Melbourne, Parkville 3052, Australia.

7 Dipartimento di Fisica dell'Università di Milano and Sezione INFN Milano, 20133 Milano, Italy

8 Laboratoire de l'Accélérateur Linéaire, Université de Paris-Sud, 91405 Orsay, France

9 Dipartimento di Fisica Nucleare e Teorica, Università di Pavia and INFN, Sezione di Pavia, Via Bassi 6, 27100 Pavia, Italy

10 Dipartimento di Fisica dell'Università di Perugia and INFN, Sezione di Perugia, via Pascoli, 06100 Perugia, Italy

11 Dipartimento di Fisica dell'Università di Pisa and INFN, Sezione di Pisa, Via Livomese, S.Piero a Grado, 56100 Pisa, Italy

12 Centre d'Etudes Nucléaires de Saclay, 91191 Gif-sur-Yvette Cedex, France

a) Now at University of Califomia, Santa Cruz, California, USA

b) Now at Lawrence Berkeley Laboratory, Berkeley, California, USA

c) Now at Istituto di Fisica, Universita di Palermo, Italy

d) Now at University of Tsukuba, Tsukuba, Ibaraki 305, Japan

e) Now at University of Stockholm, Stockholm, Sweden

f Also at Scuola Normale Superiore, Pisa, Italy

g) Now at Dipartimento di Fisica, Università di Cagliari, Italy

h) Now at Deutsches Elektronen Synchrotron, Hamburg, FRG

i) Now at Dipartimento di Fisica e INFN di Bologna, Università Bologna, Italy

j) Now at Dipartimento di Fisica dell'Università della Calabria e gruppo INFN, Cosenza, Italy

k) Now at Nuclear Physics Laboratory, University of Oxford, Oxford, UK

l) Now at CERN, Geneva, Switzerland. 


\section{INTRODUCTION}

The study of the Drell-Yan lepton pair production continuum continues to be a subject of lasting interest in hadron-hadron collisions as a function of increasing $V_{s}[1-6]$. For electron pairs lighter than the $\mathrm{Z}$ boson, the production cross section is very small, and the main experimental difficulty is to reject the large background from hadronic jets that fake the electron signature and from the production and subsequent semi-leptonic decay of heavy flavour pairs, in particular $b \bar{b}$.

This paper reports on the measurement of the electron pair production cross section, for masses below the $\mathrm{Z}$ peak, based on an event sample corresponding to an integrated luminosity of $7.1 \mathrm{pb}^{-1}$ at $\sqrt{\mathrm{s}}=630 \mathrm{GeV}$, collected by the UA2 detector during the 1988-1989 data taking period. The results are compared with theoretical calculations which include $\mathrm{O}\left(\alpha_{\mathrm{S}}^{2}\right) \mathrm{QCD}$ contributions, and the scaling properties are studied as a function of $\sqrt{ } \tau=m / \sqrt{ }$, where $m$ is the invariant mass of the lepton pair.

\section{THE UA2 APPARATUS}

A detailed description of the upgraded UA2 detector can be found in Ref. [7]. In the following only the main features relevant to this analysis are summarised.

The central calorimeter [8] covers the pseudo-rapidity region $-1<\eta<1$ over the full azimuth. Each of the 240 cells is longitudinally segmented into an electromagnetic and two hadronic compartments. In order to increase the radial space available for the central detector, the thickness of the edge cell electromagnetic compartments was reduced, thus degrading their performance. These edge cells cover the polar angles $40^{\circ}<\theta<50^{\circ}$ and $130^{\circ}<\theta<140^{\circ}$. The endcap calorimeters, covering the pseudorapidity region $1<|\eta|<3$, consist of a total of 384 cells, segmented longitudinally into an electromagnetic and a hadronic compartment.

The response of the calorimeters to muons, pions and electrons has been extensively studied in test beams. These studies have been used to calibrate the energy scale and to provide an accurate parametrization of the response to electrons as a function of the incident particle direction and impact point on the surface of the calorimeter cells, which was required for the precise determination of the $\mathrm{W}$ and $\mathrm{Z}$ masses [9]. Although the above study was performed employing electrons of $40 \mathrm{GeV}$ incident energy, additional data were also taken with electrons of 10 and $20 \mathrm{GeV}$ which are more relevant to the present analysis. The energy resolution for electromagnetic showers is typically $\sigma_{\mathrm{E}} \sim 16 \% \sqrt{\mathrm{E}}(\mathrm{E}$ in $\mathrm{GeV})$. 
The central detector consists of :

- Two arrays of silicon pad detectors (SI), at radii of $3.5 \mathrm{~cm}$ and $14.5 \mathrm{~cm}$. These are used for tracking and ionization measurements [10].

- A cylindrical drift chamber (JVD), located between the two silicon arrays and used for track reconstruction [11].

- Two transition radiation detectors, each consisting of a radiator and an X-ray detector, located outside the silicon arrays. They can be used to improve the electron identification [12].

- A scintillating fibre detector (SFD), consisting of fibres arranged in 18 layers followed by a 1.5 radiation length thick lead converter and a further 6 layers which are used to localize electromagnetic showers initiated in the converter ("preshower" detector) [13]. The SFD is the outer component of the central detector.

In the forward region, in front of the endcap calorimeters, there is a tracking detector [14], consisting of proportional tubes and including a 2 radiation length thick converter, which provides both tracking and preshower information.

For the analysis presented here the data were obtained from the electron pair trigger [15], where at least two electromagnetic clusters in the central calorimeter were required to have transverse energies $\left(\mathrm{E}_{\mathrm{T}}\right)$ above $5.5 \mathrm{GeV}$ and an azimuthal separation of at least $60^{\circ}$.

The luminosity was measured by means of a telescope of scintillator counters at small angles with respect to the beams. The algorithm used to calculate the integrated luminosity took into account the effects of multiple interactions in the same bunch crossing, and the acceptance of this system was measured using minimum bias data [7]. The computed total luminosity accumulated for the combined 1988 and 1989 data taking periods was $7.4 \mathrm{pb}^{-1}$. After removing the data where not all of the detectors required for the present analysis were operational, the useful luminosity was $7.1 \pm 0.4 \mathrm{pb}^{-1}$, where the error arises from the uncertainty on the acceptance of the telescopes (2.3\%) [7], and a $4.7 \%$ contribution from the uncertainty on the $\bar{p} p$ total cross section [16].

\section{ELECTRON SELECTION CRITERIA AND EFFICIENCIES}

In the present study, only electron candidates in the central calorimeter are considered. For the final event sample, the criteria used to select an electron candidate require an electromagnetic cluster (CAL cut) to be associated with a reconstructed track 
(TRK cut) and a preshower signal (PS cut). In addition, the lateral and longitudinal profiles of the shower were required to be consistent with those expected for a single isolated electron incident along the track direction as determined from test beam data. From the observed and expected quantities and their estimated errors, a $\chi^{2}$ test for the electron hypothesis was defined $\left(\mathrm{P}\left(\chi^{2}\right)\right.$ cut).

The directions of the charged particle tracks and the position of the event vertex along the beam axis were reconstructed using the SFD in conjunction with the SI and JVD detectors. The reconstructed event vertex was required to lie within $250 \mathrm{~mm}$ of the detector center. The corresponding efficiency was measured to be $\varepsilon_{\mathrm{V}}=94.3 \pm 0.5 \%$.

The tracking and preshower sections of the SFD were used to match the trajectories of candidate central electron tracks with the position of electromagnetic showers with a resolution of $\sigma_{\mathrm{r} \phi}=0.4 \mathrm{~mm}$ in the $\mathrm{r}-\phi$ plane (perpendicular to the beam axis) and $\sigma_{\mathrm{Z}}=1.1 \mathrm{~mm}$ along the beam direction, measured using a sample of electrons from $\mathrm{W} \rightarrow$ ev decays. The quality of the track-preshower match was defined by the variable $\mathrm{d}_{\sigma}^{2}=\left(\Delta_{\mathrm{r} \phi} / \sigma_{\mathrm{r} \phi}\right)^{2}+\left(\Delta_{\mathrm{Z}} / \sigma_{\mathrm{Z}}\right)^{2}$, where $\Delta_{\mathrm{r} \phi}$ and $\Delta_{\mathrm{Z}}$ are the measured displacements between the track and preshower positions. The accidental association of showers induced by photons with charged tracks gives, in general, large values of $d_{\sigma}^{2}$. Electron candidates were required to have $\mathrm{d}_{\sigma}^{2}<25$.

The efficiency of the CAL cut was determined from test beam data, whereas the efficiencies of the other cuts were measured from the data themselves. The method employed for the latter starts from the sample of 165571 electromagnetic cluster pairs (referred to as EM hereafter), to which the electron selection cuts are applied. The events are classified in three categories depending on whether none, one or both clusters satisfy the electron selection criteria respectively. In an analogous procedure to Ref. [17], a set of equations can be established, that relate the observed number of events to the contributions :

$$
N_{i j}=\left(2 \varepsilon_{i} \varepsilon_{j}-\varepsilon_{j}^{2}\right) \times S+\left(\frac{2}{R_{i} R_{j}}-\frac{1}{R_{j}^{2}}\right) \times B
$$

where $N_{i j}$ is the number of events which satisfy the selection $i$ and $j$ (where $j$ includes $i$ ), $S$ is the number of signal events (genuine electron pairs) and $B$ is the number of background events (predominantly from hadronic jets that fake the electron signature), $\varepsilon_{\mathrm{i}}$ is the efficiency for electrons and $R_{i}$ the rejection against hadrons for the selection $i$. These equations are solved numerically in order to obtain an estimate of the efficiencies. 
The combined efficiency of the above cuts is measured to be

$$
\varepsilon_{\mathrm{e}}=\varepsilon_{\mathrm{CAL}} \times \varepsilon_{\mathrm{TRK}} \times \varepsilon_{\mathrm{PS}} \times \varepsilon_{\mathrm{P}\left(\chi^{2}\right)}=(56.1 \pm 3.6) \%,
$$

for the non-edge cells, and

$$
\varepsilon_{\mathrm{e}}=(46.2 \pm 5.0) \%
$$

for the edge cells of the central calorimeter.

These efficiencies are slightly lower, as expected, than the values obtained from a sample of $\mathrm{W} \rightarrow \mathrm{ev}$ decays [7], where the average energy of the electron is $40 \mathrm{GeV}$.

\section{DRELL-YAN SIGNAL AND BACKGROUND ESTIMATION}

Applying the electron selection criteria described in section 3 to both EM clusters in the event yields a sample of 124 electron pair candidates in the mass interval $10<\mathrm{m}$ $<70 \mathrm{GeV}$. The mass spectrum for these events is shown in Fig. 1. In addition, Fig. 1 also contains 70 events with a mass larger than $70 \mathrm{GeV}$. These are identified as $\mathrm{Z} \rightarrow \mathrm{e}^{+} \mathrm{e}^{-}$ decays, where the observed number of events is consistent with the published UA2 value of the $\mathrm{Z}$ production cross section [7].

\subsection{Evaluation of the background}

The two principal contributions to the event sample are the genuine electron pairs from the Drell-Yan process, $\overline{\mathrm{p}} \mathrm{p} \rightarrow \gamma^{*} / \mathrm{Z} \rightarrow \mathrm{e}^{+} \mathrm{e}^{-}+\mathrm{X}$, and fake electrons from QCD processes (where hadronic jets fake the electron signature). This "QCD background" can be estimated using the data themselves. This is achieved by a procedure analogous to that used to determine the efficiencies (section 3), yielding $27.4 \pm 2.8$ events, where the error includes the systematic uncertainties due to the procedure used for the estimate.

The PYTHIA Monte Carlo programme [18] was used to estimate the background in the event sample arising from $b \bar{b}$ production with the subsequent semielectronic decay of the $b$ quark. The $b \bar{b}$ events are generated according to the subprocesses $q \bar{q} \rightarrow b \bar{b}$ and $\mathrm{gg} \rightarrow \mathrm{b} \overline{\mathrm{b}}$. The electrons produced in the decay $\mathrm{b} \rightarrow \mathrm{cev}$, are, in general, not isolated, leading to a significantly lower efficiency for the electron selection criteria. This was studied by Monte Carlo simulations, using a similar procedure to that described in the context of the search for top quark production in UA2 [19]. The Monte Carlo calculation 
included a full simulation of the calorimeter response. In order to normalize the Monte Carlo events to the integrated luminosity, the theoretical value of the $b \bar{b}$ production cross section (including the appropriate kinematical cuts) $\sigma(p \bar{p} \rightarrow b \bar{b}+X)=4.0_{-1.5}^{+2.5} \mu b[20]$ is used. The number of background events thus obtained is $7.4 \pm 3.7$, where the dominant error is the $\sim 50 \%$ uncertainty in the $b \vec{b}$ production cross section.

Finally, the background contribution to the electron pair sample from the process $\mathrm{q} \overline{\mathrm{q}} \rightarrow \gamma^{*} / \mathrm{Z} \rightarrow \tau^{+} \tau^{-}$, followed by $\tau \rightarrow \mathrm{eVV} \tau$ is estimated to be $\sim 0.5$ events.

\subsection{The Drell-Yan cross section}

The PYTHIA Monte Carlo programme [18] was used in order to calculate the detector acceptance, which is required to compute the cross section. The events are generated according to the subprocess $\mathrm{q} \overline{\mathrm{q}} \rightarrow \gamma^{*} / \mathrm{Z}^{0} \rightarrow \mathrm{e}^{+} \mathrm{e}^{-}$, which includes $\mathrm{O}\left(\alpha_{\mathrm{s}}\right)$ contributions, with the structure functions of Ref. [21]. The simulation programme takes into account the geometrical acceptance of the detector and the effect of the trigger threshold, as well as the electron energy resolution. The acceptance as a function of the electron pair mass is shown in Fig. 2. The uncertainty on the acceptance is dominated by the choice of the structure function sets.

The measured cross section is determined from the relation

$$
\sigma=\frac{\mathrm{N}-\mathrm{B}}{\varepsilon \mathrm{A} \mathscr{L}}
$$

where $\mathrm{N}$ is the observed number of events, $\mathrm{B}$ is the sum of all background processes, $\mathrm{A}$ is the acceptance of the geometrical and kinematic selection, $\varepsilon$ is the overall electron detection efficiency, and $\mathscr{L}$ is the integrated luminosity. The cross section calculated from the events with both electrons in the non-edge cells, and the events with one electron in the edge cell and the other in the non-edge cell of the central calorimeter is :

$$
\sigma=405 \pm 51 \text { (stat.) } \pm 84 \text { (syst.) pb }
$$

in the mass interval $10<\mathrm{m}<70 \mathrm{GeV}$. The systematic error reflects the uncertainties in the quantities used to calculate the cross section (i.e. acceptance, luminosity and the various efficiencies), as well as the systematic uncertainties on the QCD and $b \bar{b}$ backgrounds. These results are in agreement, within errors, with previous measurements at the CERN p pollider [5,6]. The differential cross section $(\mathrm{d} \sigma / \mathrm{dm})$ for four mass intervals is shown in Fig. 3. 
Until recently, the cross section for lepton pair production had been calculated up to order $\alpha_{\mathrm{s}}$. Presently, calculations exist that go beyond $\mathrm{O}\left(\alpha_{\mathrm{S}}\right)$ and compute the dominant second order corrections to the lepton pair production [22], with the $\gamma^{*} / Z$ interference term of the propagator. A complete calculation has been used in the context of the $\mathrm{W}$ and $\mathrm{Z}$ production cross section, and a detailed discussion can be found in Ref. [7]. The main uncertainties in the theoretical calculation arise from the parton distribution functions, and the evolution of $\alpha_{s}$ with $Q^{2}$, where the $Q^{2}$ scale is taken to be the invariant mass of the lepton pair. It is important to note that the variations obtained in the calculation, e.g. for a lepton pair effective mass of $30 \mathrm{GeV}$, are $\sim 15 \%$, depending on the values of the parameters, which illustrates the size of the theoretical uncertainties. The results of the calculation as a function of the lepton pair mass are shown in Fig. 3. Despite the overall good agreement with the data, the present statistical and systematic errors cannot be used for a quantitative conclusion regarding the agreement with higher order $\alpha_{S}$ calculations.

Figure 4 shows a comparison of the scaled differential cross section, $\mathrm{m}^{3} \mathrm{~d}^{2} \sigma / \mathrm{dmdy}$ at $y=0$, where $y$ is the rapidity of the electron pair, with previous measurements performed at lower energies [1-4], versus the scaling variable $\sqrt{ } \tau=\mathrm{m} / \mathrm{V}$ s. The results are consistent with scaling albeit with large errors.

\section{CONCLUSIONS}

A measurement of Drell-Yan electron pair production cross section from a sample of events obtained with the UA2 detector at the CERN $\bar{p}$ p Collider at $\sqrt{s}=630 \mathrm{GeV}$, yields $\sigma=405 \pm 51$ (stat.) \pm 84 (syst.) pb in the mass interval $10<\mathrm{m}<70 \mathrm{GeV}$.

The results are consistent with recent theoretical calculations including $O\left(\alpha_{\mathrm{S}}^{2}\right)$ contributions. Approximate scaling in the variable $\sqrt{ } \tau=\mathrm{m} / \sqrt{s}_{\mathrm{s}}$ is observed when comparing these results with previous measurements. 


\section{ACKNOWLEDGEMENTS}

We gratefully acknowledge the technical staff of the institutes collaborating in UA2 for their continuous support. We thank T. Matsuura for helpful discussions and for providing calculations for the cross section in the form of computer programmes.

Financial support from the Schweizerischen Nationalfonds zur Förderung der Wissenschaftlichen Forschung to the Bern group, from the UK Science and Engineering Research Council to the Cambridge group, from the Bundesministerium für Forschung und Technologie to the Dortmund and Heidelberg groups, from the Australian Research Council, the CRA Pty Ltd, and the Victorian Education Foundation to the Melbourne group, from the Institut National de Physique Nucléaire et de Physique des Particules to the Orsay group, from the Istituto Nazionale di Fisica Nucleare to the Milano, Pavia, Perugia and Pisa groups and from the Institut de Recherche Fondamentale (CEA) to the Saclay group are acknowledged. 


\section{REFERENCES}

[1] J. Badier et al., Z. Phys. C26 (1985) 489.

[2] A.G. Clark et al., Nucl. Phys. B142 (1978) 29.

[3] A.S. Ito et al., Phys. Rev. D23 (1981) 604.

[4] D.M. Kaplan et al., Phys. Rev. Lett. 40 (1978) 435.

[5] P. Bagnaia et al., Phys. Lett. B154 (1985) 338.

[6] C. Albajar et al., Phys. Lett. B186 (1987) 237.

[7] J. Alitti et al., Z. Phys. C47 (1990) 11;

J. Alitti et al., A Measurement of the $\mathrm{W}$ and $\mathrm{Z}$ Production Cross Section and a Determination of $\Gamma_{W}$ at the CERN $\bar{p}$ p Collider, CERN-PPE/91-162, submitted to Phys. Lett.

[8] A. Beer et al., Nucl. Instrum. Methods A224 (1984) 360.

[9] J. Alitti et al., Phys. Lett. B241 (1990) 150;

J. Alitti et al., An Improved Determination of the Ratio of $\mathrm{W}$ and $\mathrm{Z}$ masses at the CERN p̄p Collider, CERN-PPE/91-163, submitted to Phys. Lett.

[10] R. Ansari et al., Nucl. Instrum. Methods A279 (1989) 388.

[11] F. Bosi et al., Nucl. Instrum. Methods A283 (1989) 532.

[12] R. Ansari et al., Nucl. Instrum. Methods A263 (1988) 51.

[13] R. Ansorge et al., Nucl. Instrum. Methods A265 (1988) 33.

[14] K. Borer et al., Nucl. Instrum. Methods A286 (1990) 128.

[15] K. Einsweiler et al., Proc. Int. Conf. on the Impact of Digitial Microelectronics and Microprocessors on Particle Physics (1988) 247;

S. Stapnes, ibid., 254. 
[16] M. Bozzo et al., Phys. Lett. B147 (1984) 392.

[17] T. Åkesson et al., Phys. Lett. B238 (1990) 442.

[18] H.U. Bengtsson and Torbjörn Sjöstrand, The Lund Monte Carlo for Hadronic Processes - PYTHIA Version 4.8. LU TP 87-3, UCLA-87-001 (1987).

[19] T. Åkesson et al., Z. Phys. C46 (1990) 179.

[20] P. Nason et al., ETH-PT/89-2, Fermilab-PUB-89/91-T, BNL-42398 (1989).

[21] M. Diemoz et al., Z. Phys. C39 (1988) 21.

[22] T. Matsuura, Higher order corrections to the Drell-Yan process. Ph. D. Thesis, University of Leiden, 1989; and private communications;

R. Hamberg, W.L. van Neerven and T. Matsuura, Nucl. Phys. B359 (1991) 343. 


\section{FIGURE CAPTIONS}

Fig. 1 Distribution of the electron pair mass, (m), for the selected events.

Fig. 2 The detector acceptance for Drell-Yan electron pairs as a function of the mass (m).

Fig. 3 The differential cross section (do/dm) as a function of the mass (m). The results of the theoretical prediction are show for the Born term (dotted line), $O\left(\alpha_{\mathrm{s}}\right)$ (dashed line) and $O\left(\alpha_{\mathrm{S}}^{2}\right)$ (solid line). The error bars represent the statistical uncertainty (solid line) and the systematic uncertainty (dashed line) on the experimental points.

Fig. 4 A comparison of the scaled Drell-Yan lepton pair production cross section $\left(\mathrm{m}^{3} \mathrm{~d}^{2} \sigma /\right.$ dmdy at $\left.\mathrm{y}=0\right)$ as a function of the scaling variable $\sqrt{\tau}=\mathrm{m} / \sqrt{\mathrm{s}}$. 


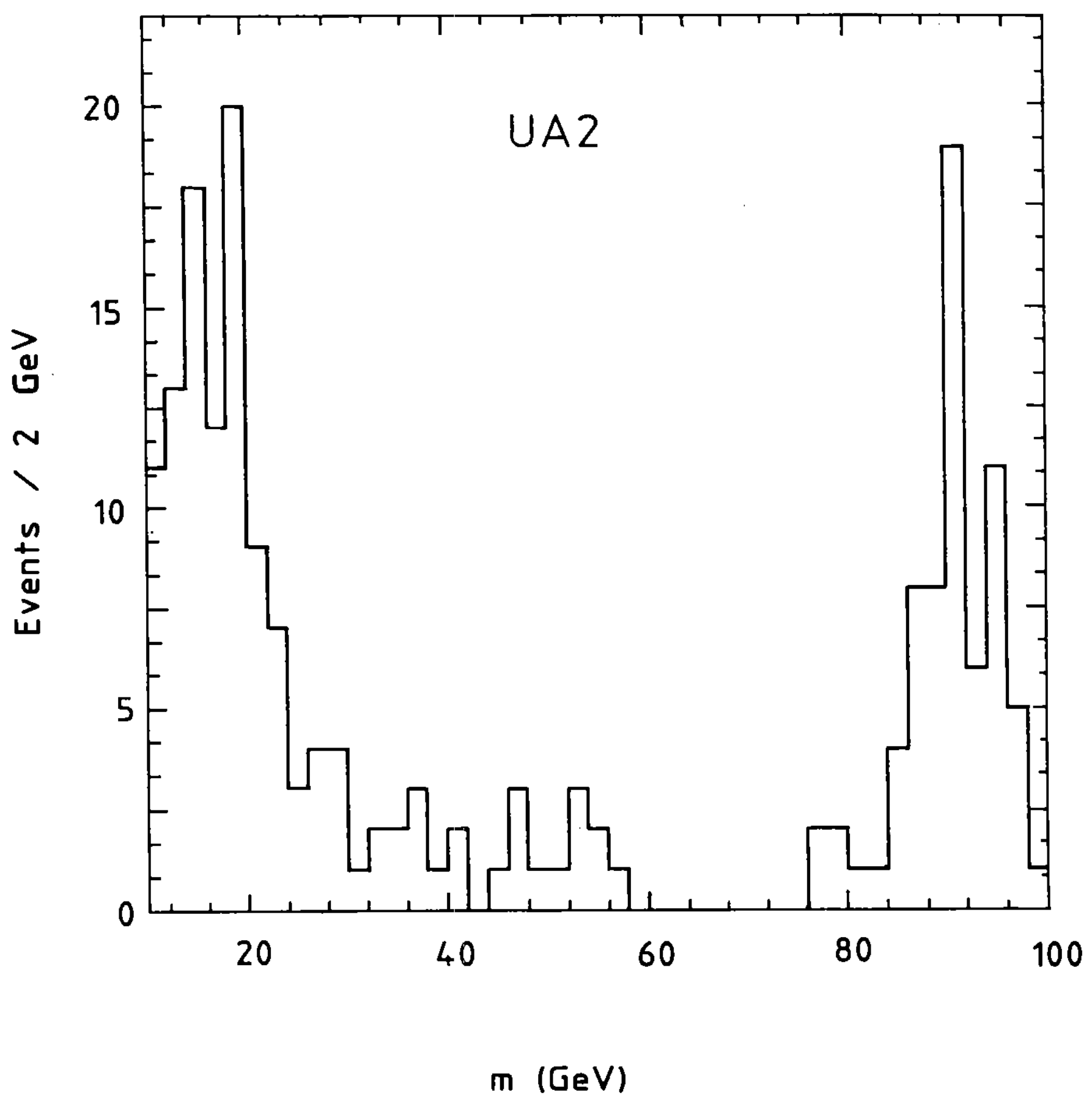

Fig. 1 


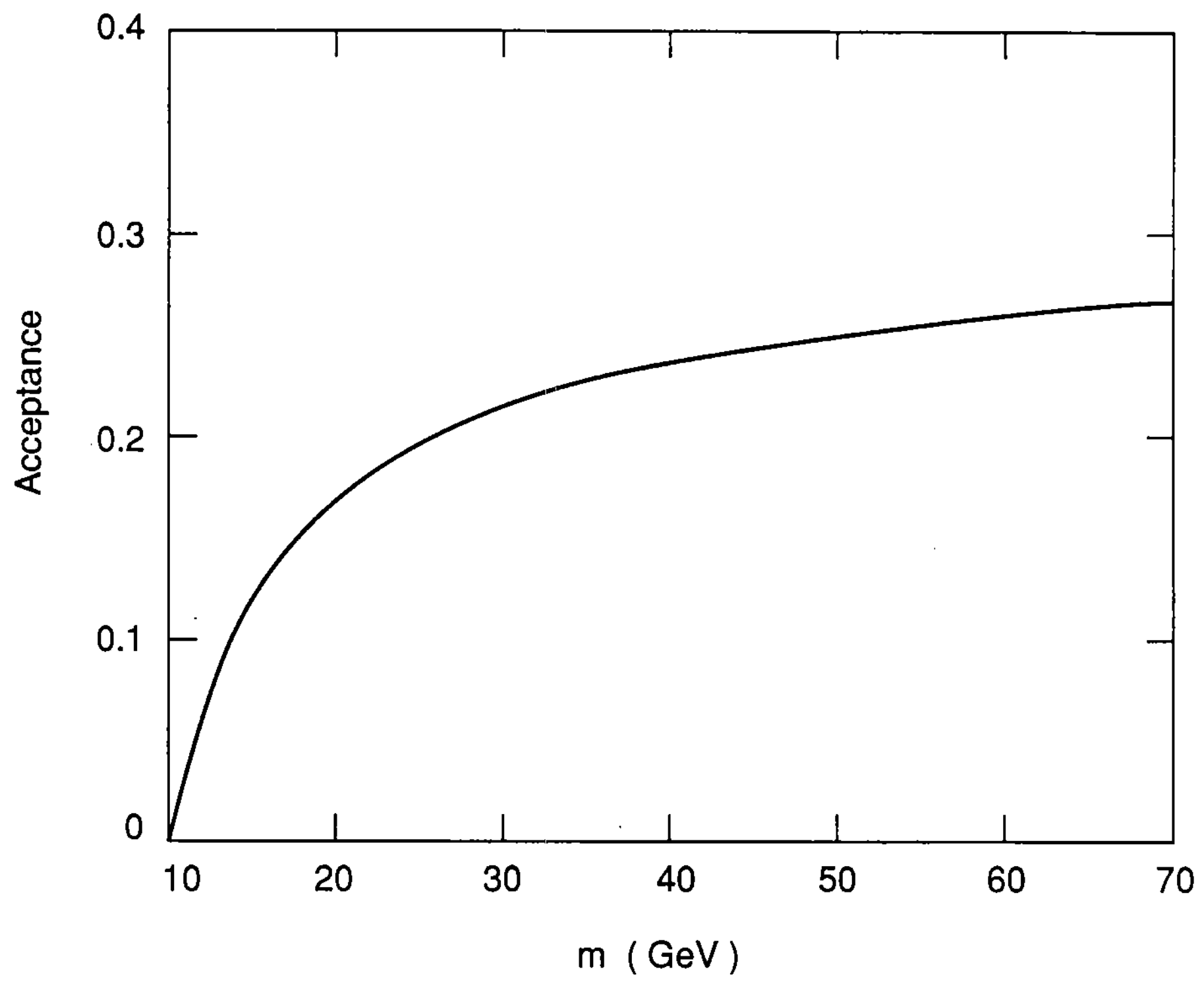

Fig. 2 


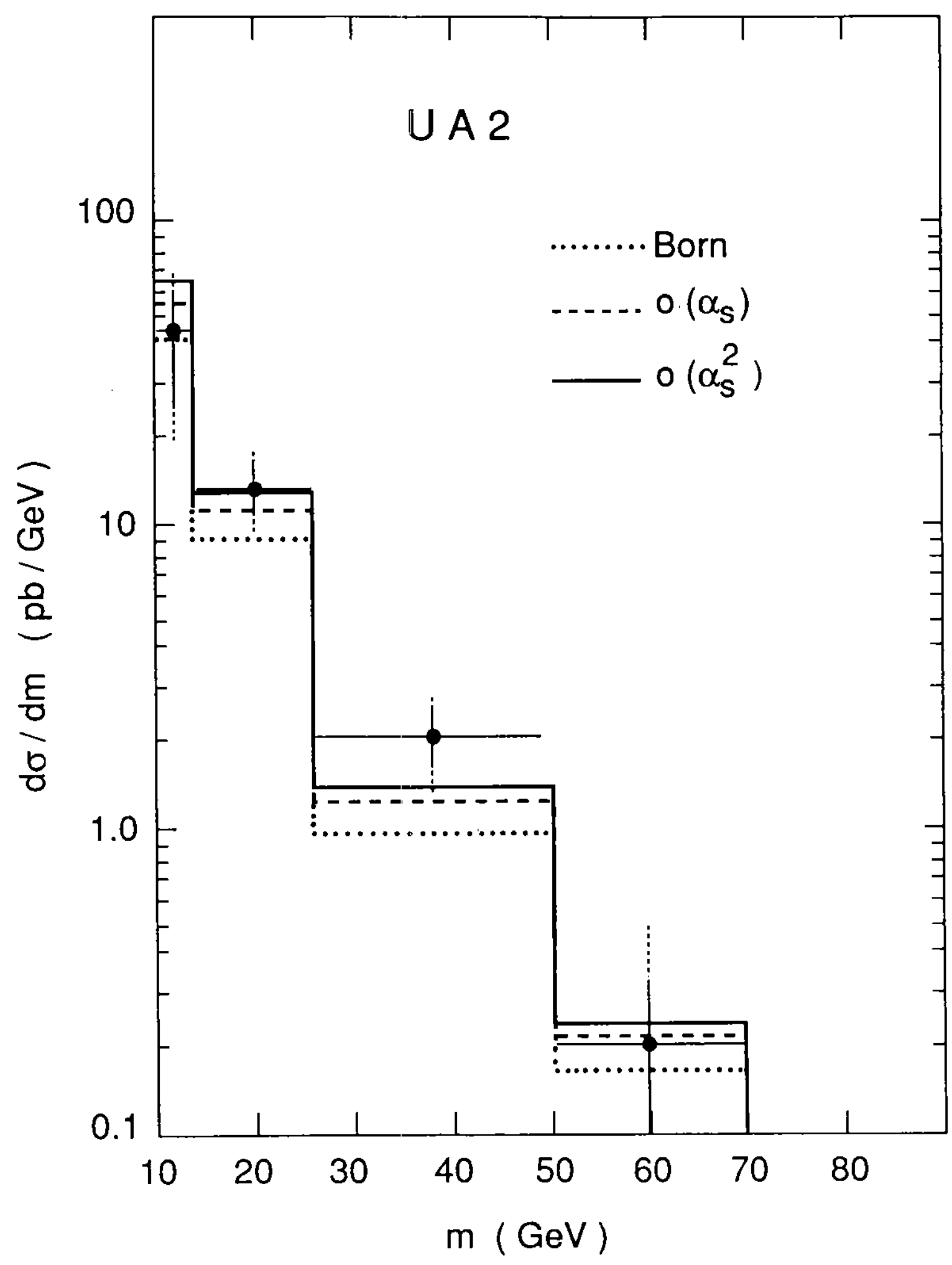

Fig. 3 


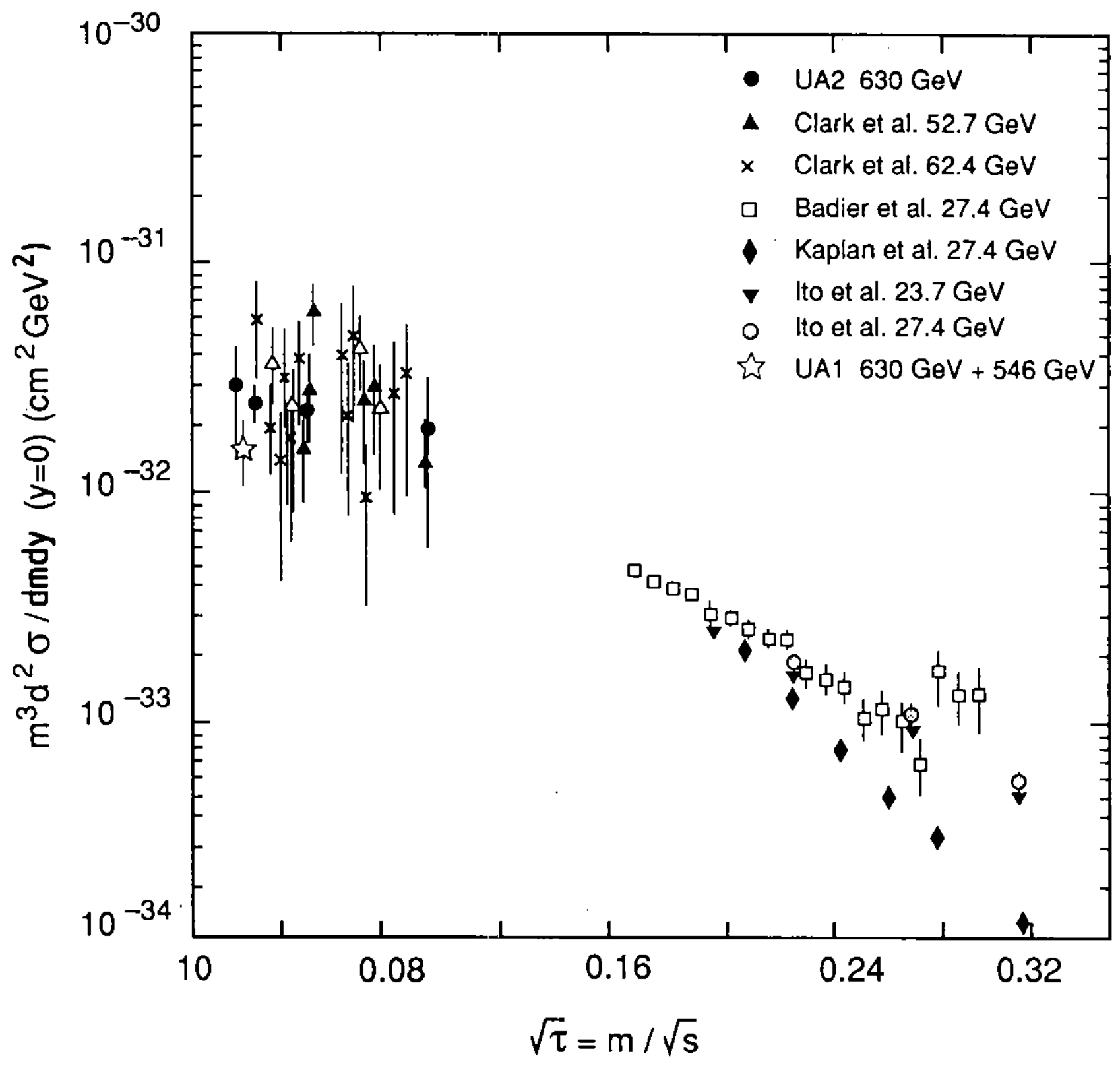

Fig. 4 